\title{
Metabolic and hematological disorders of cytoreductive surgery with hyperthermic intraperitoneal chemotherapy (HIPEC)
}

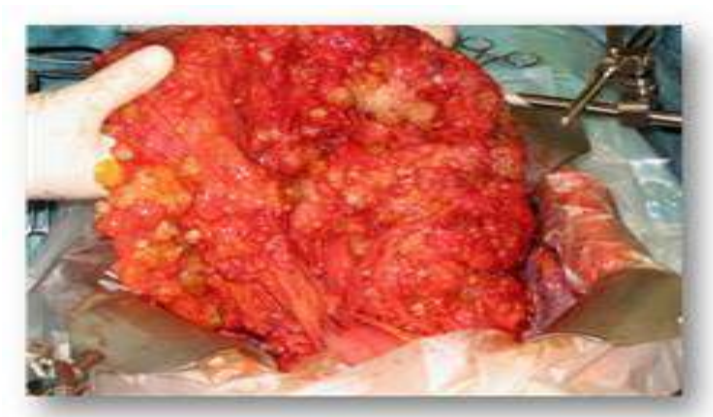

Gómez G, Ferrer A, Esteve N, Fabian D, Melero C, Mora LC.

Son Espases University Hospital

IdISPA, Health Research Institute of Palma.

Cytoreductive surgery (CRS) with Hyperthermic Intraperitoneal Chemotherapy (HIPEC) produces important metabolic and hematological disorders during the entire perioperative period ${ }^{1}$.

We present the evolution of metabolic and hematological parameters of patients who underwent CRS with HIPEC.

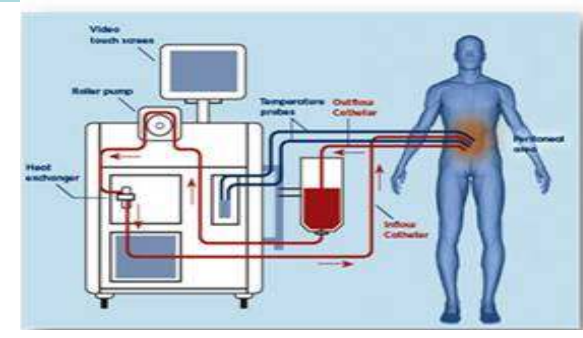

Methods

A prospective descriptive study was conducted of consecutive patients with Peritoneal Carcinomatosis (PC) who underwent CRS with HIPEC between March 2014 and February 2015. We applied an anaesthesia protocol and goaldirected hemodynamic and metabolic therapy.

Results

The $\mathrm{pH}$ decreased from $7.42[7.32$ 7.57] to 7.28 [7.26 - 7.30] during HIPEC, which is not associated with increased Cl-. Our fluid therapy protocol includes the use of balanced fluids. $75 \%$ women

$58 \%$ Ovarian $\mathrm{Ca}$.

$33 \%$ CRS

Lactate values increased during the HIPEC up to $3.38 \mathrm{mmol} \cdot \mathrm{L}^{-1}$ [2.81 - 4.0], which normalized on the 1st postoperative day without any treatment.
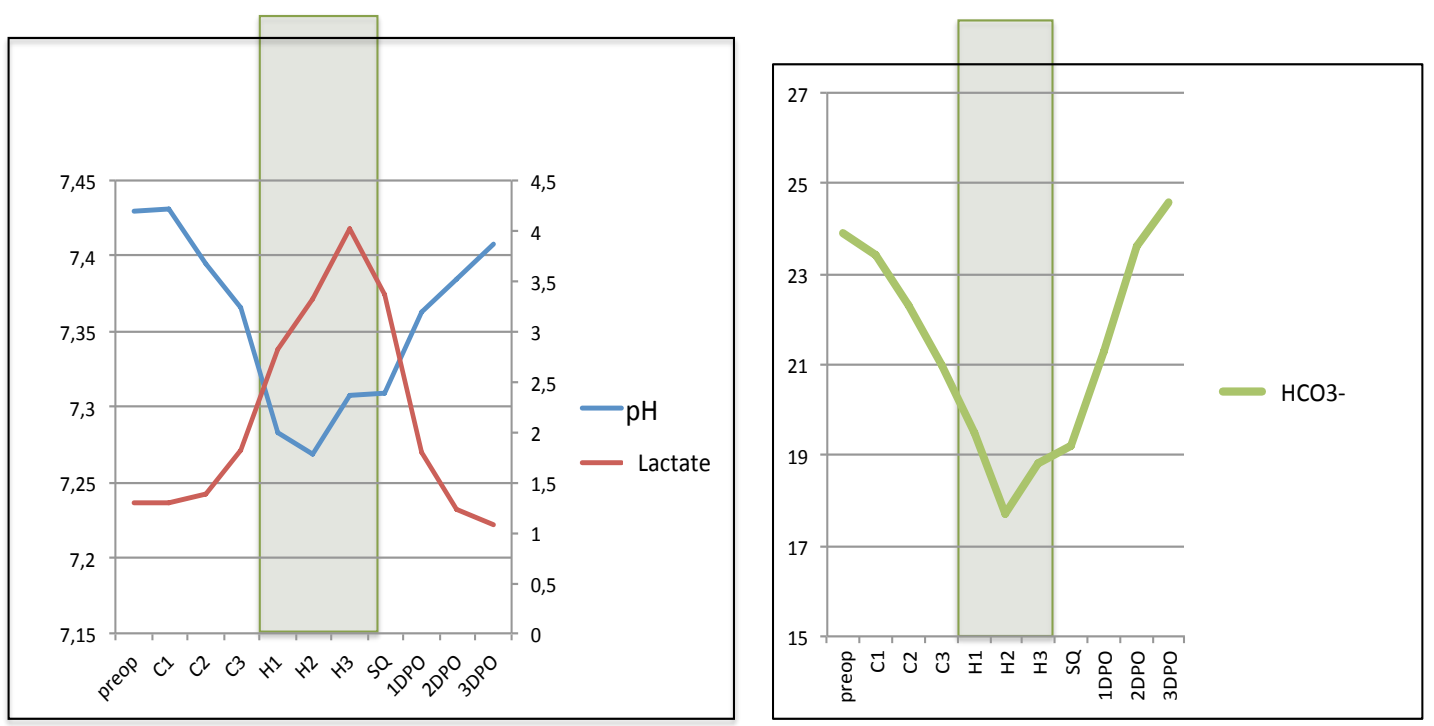

Hyperglicemia, 396 mg/dl [379-426], occurred during HIPEC in the first 12 patients when using glucose $5 \%$ as solvent of cytostatic.

The hyperglicemia dissapeared after using glucose $1,25 \%$ as solvent.
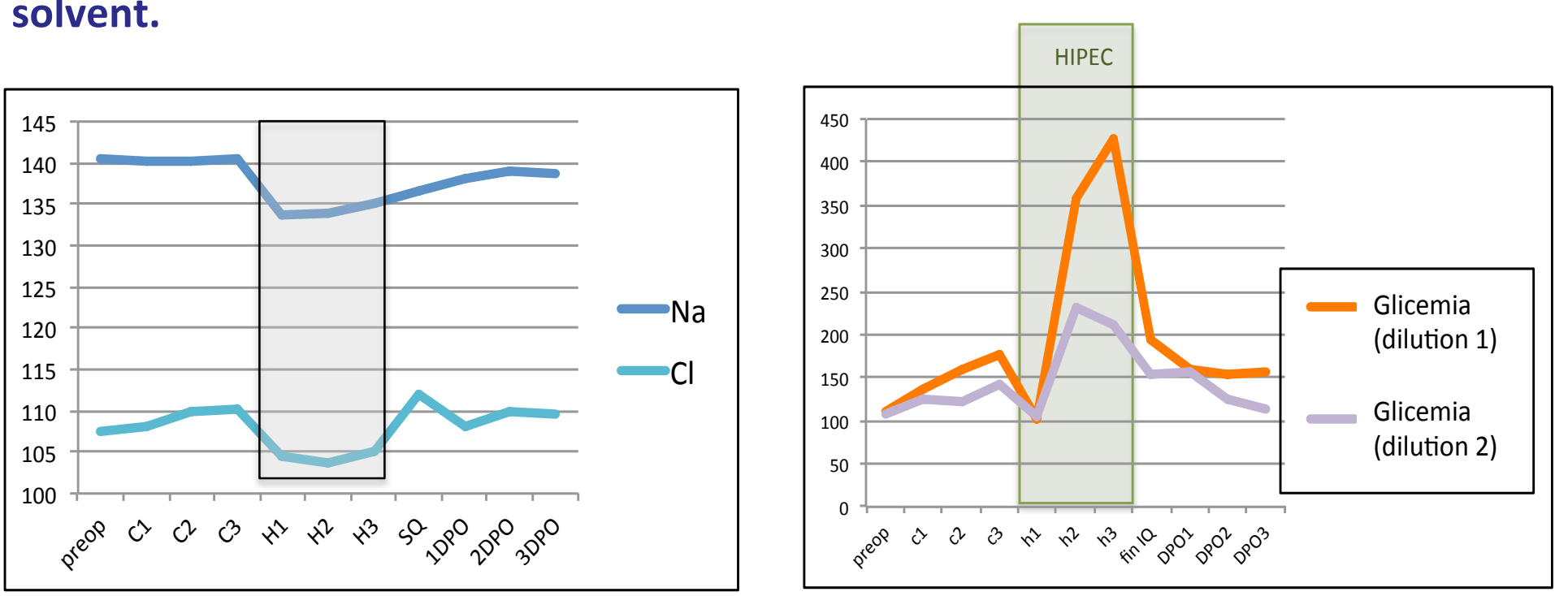

Lower Platelet and higher INR means values were recorded the second postoperative day, being $159 \cdot 10^{9} \mathrm{I}^{-1}$ [73-341] and 1.41 [1.21 - 1.94], respectively, regaining normal values without treatment .

$75 \%$ of patients had hypophosphatemia, $92 \%$ hypomagnesemia and $46 \%$ hypocalcemia. All patients showed hypoalbuminemia.
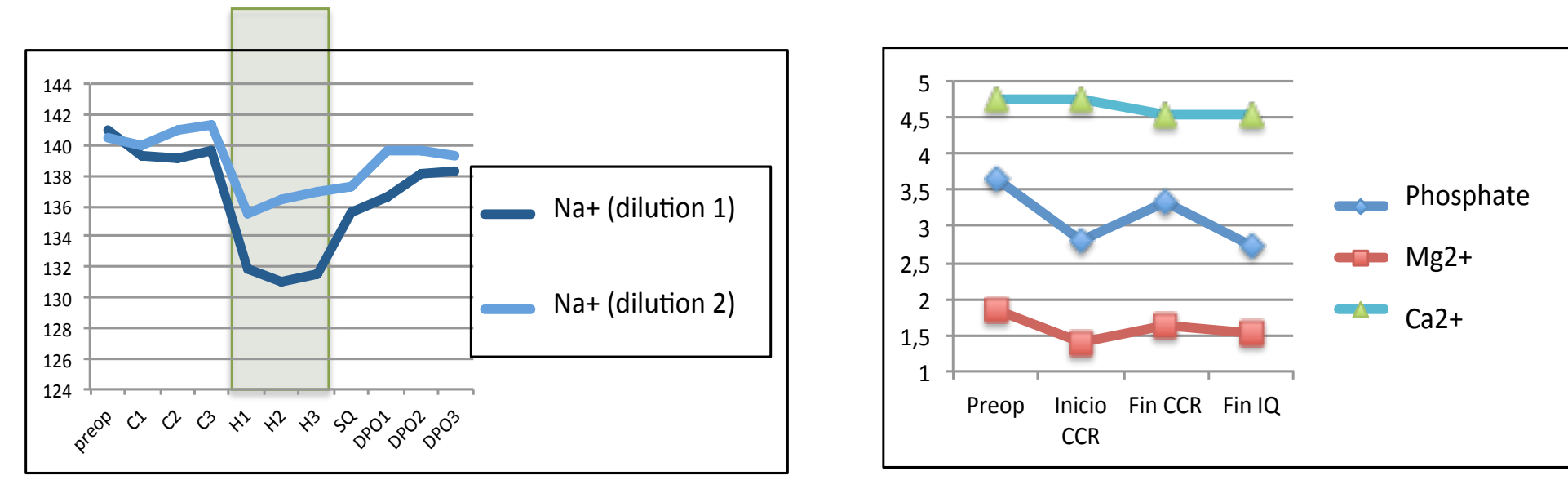

$29 \%$ of patients were transfused during surgery, with an average of 2 red blood cells pack per patient. There were no differences of hemoglobin levels at the beginning of the CRS between transfused or not transfused patients 9.4 (8.1-10.7) vs $10.7(9.7-11.3)(p=0.114)$.
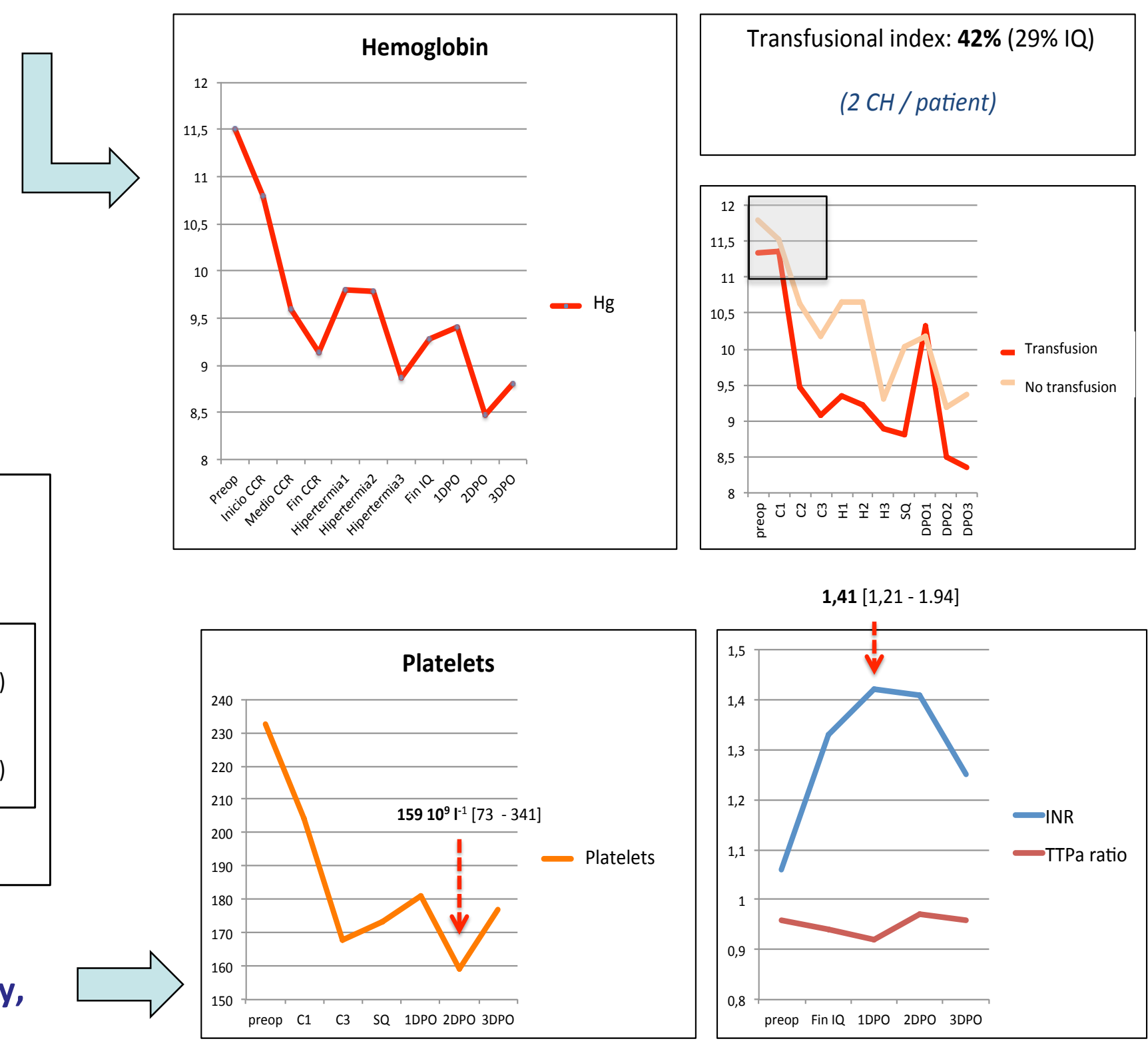

\section{Conclusions}

In line with other studies², metabolic non-hipercloremic acidosis is self-limiting and does not require treatement. Balanced fluid therapy should be used to prevent an increase in acidosis.

The use of Cytostatic solvent glucose $1,25 \%$ avoid the hyperglicemia and insulin infusion ${ }^{3}$. Hypomagnesemia, hypocalcemia and hypoalbuminemia usually requires systematic replacement.

Preoperative treatment of the anemia could be considered. Coagulation abnormalities had normalized after the third day in most patients. without treatment.

Webb CA, Weyker PD, Moitra VK, Raker RK. An overview of cytoreductive surgery and hyperthermic intraperitoneal chemoperfusion for the anesthesiologist. Anesth Analg. 2013 Apr;116(4):924-31.

Pascual J, Sanchez S, Gonzalez F, Villarejo P, López de la Manzanara C, Haya J, Padilla D, Martin J. Security and efficiency of a closed-system, turbulent-flow circuit for HIPEC after cytoreductive ovarían surgery: perioperative outputs. Arch Gynecol Obstet. 2014; 290:121-129.

Raytis J.L and Lew, M.W. Hyperthermic Intraperitoneal Chemotherapy (HIPEC) and Intraoperative Electrolyte Disturbances -Implications for Anesthetic Management Open Journal of Anesthesiology 2014; 4: 240-243. 